\title{
Factores de riesgo de bajo peso al nacer
}

\author{
Marcelo Bortman ${ }^{1}$
}

RESUMEN El bajo peso al nacer (BPN) es el principal factor determinante conocido de la mortalidad infantil. Pese a la notable reducción de las tasas de mortalidad infantil y al aumento de las tasas de supervivencia de niños con BPN, en Neuquén, Argentina, no se han observado descensos notables de las tasas de BPN. El propósito de este estudio fue conocer los factores de riesgo de BPN, su frecuencia en la población y el papel de la atención prenatal en su prevención, y desarrollar una escala de riesgo que permita identificar a las mujeres en mayor riesgo de dar a luz un niño de bajo peso. Con tal propósito se realizó un estudio transversal en el cual se utilizó $50 \%$ de los datos del Sistema Informático Perinatal correspondientes al período 1988-1995 y procedentes de los 29 hospitales de la provincia de Neuquén (46 171 nacimientos). Se analizaron la distribución del peso y la frecuencia de los posibles factores de riesgo de BPN. La asociación entre estos factores y el BPN se analizó mediante un modelo de regresión logística. Con los resultados obtenidos se desarrolló una escala aditiva, que fue validada con el $50 \%$ restante de los registros de nacimientos. La razón de posibilidades $(R P)$ más elevada correspondió a la falta de atención prenatal ( $R P=8,78$; IC95\%: 6,7 a 11,4). Las $R P$ asociadas con una atención prenatal inadecuada, una primera consulta prenatal tardía, preeclampsia o eclampsia, hemorragia o anomalias de la placenta o sus membranas e historia de un hijo previo con BPN fueron mayores de 2,0. El riesgo de tener hijos con BPN también fue más alto en las mujeres mayores de 40 años, las menores de 20, las solteras, las fumadoras, aquellas cuyo intervalo intergenésico fue menor de 18 meses y las que tenían un indice de masa corporal menor de 20. Por último, se encontró una relación lineal directa entre el puntaje en la escala y el riesgo de tener un hijo con BPN.

Aunque hasta fines del siglo XIX no se comenzó a pesar a los recién nacidos, la observación de que la probabilidad de sobrevivir estaba estrechamente relacionada con su peso al nacer se conocía desde algunos siglos antes. El bajo peso al nacer (BPN) - definido por un grupo de expertos de la OMS $(1,2)$ y en la Clasificación internacional de enfermedades (3) como un peso menor de 2500 gramos en el momento de nacer- es el principal factor determinante de la mortalidad infantil y, especialmente, de la neonatal (5-8). El BPN

\footnotetext{
1 Subsecretaría de Salud, Dirección de Información y Programación, Neuquén, Argentina. Dirección postal: Fotheringham 121, 8300 Neuquén, Argentina.
}

aumenta varias veces el riesgo de morbilidad neonatal e infantil $(4,5)$, causa trastornos familiares y sobrecarga los presupuestos de los servicios de cuidados intensivos y neonatales especiales (9-11). El BPN también se asocia estrechamente con alteraciones del desarrollo infantil y algunos informes sugieren que más de $50 \%$ de la morbilidad neurológica crónica es atribuible a este trastorno (12). Recientemente el BPN se ha asociado con irregularidades del crecimiento fetal y con algunos trastornos del adulto, tales como la enfermedad coronaria (13).

La distribución del peso al nacer sigue una curva normal con una curtosis hacia los valores bajos debido a un exceso de niños con peso bajo y muy bajo al nacer, comparados con el número de niños de peso alto. Este exceso es especialmente perceptible en las poblaciones de países en desarro1lo. Wilcox y Russell sugirieron en 1986 que esta distribución puede fraccionarse al menos en dos sectores simétricamente distribuidos. El sector mayor incluye a los niños de peso normal al nacer; el otro, a los más pequeños. Este segundo grupo crítico parece verse afectado por una serie de factores definibles y ello tiene repercusiones importantes en el desarrollo de estrategias de prevención (14).

En 1982, varios investigadores notificaron que la proporción de BPN en el mundo oscilaba entre 3 y 43\%. En los países desarrollados, la incidencia de 
BPN se encontraba entre 3,6 y 7,4\%, mientras que en países de Asia Sudoriental dicha incidencia variaba de 20 a $43 \%$ (15). Los porcentajes de muertes infantiles atribuibles a BPN en los países en desarrollo están subestimados como consecuencia de la alta prevalencia de enfermedades infecciosas notificada en ellos. Si se tiene en cuenta que más de $90 \%$ de los niños con bajo peso nacen en esos países, es fácil comprender la influencia que en ellos ejerce el BPN en la mortalidad infantil. Por otro lado, la incidencia mundial de BPN en 1990 fue de 17\% (16). Consecuentemente, la meta de la OMS de reducir en $10 \%$ la incidencia de BPN está todavía muy lejos de ser alcanzada.

El BPN es consecuencia de un crecimiento intrauterino inadecuado, de un período gestacional demasiado corto, o de la combinación de ambas alteraciones. Por ello, cabe esperar que los factores relacionados con el BPN representen una confluencia de las causas básicas del parto pretérmino y del retraso del crecimiento intrauterino. Pese a los continuos adelantos médicos, el conocimiento de las causas básicas de estos trastornos sigue siendo parcial. Aunque muchos de los factores de riesgo conocidos solo pueden considerarse marcadores de las causas verdaderas y subyacentes, pueden ser muy útiles para identificar grupos de riesgo en la población. En una revisión de la literatura se indicó que la desnutrición materna constituye un factor causal del crecimiento fetal inadecuado (17).

Los factores asociados con el BPN pueden clasificarse en cuatro grupos generales: demográficos, médicos, conductuales y ambientales. Como se observa en el cuadro 1, los factores médicos pueden subdividirse a su vez en los que están presentes antes de la gestación y en los que se manifiestan en su transcurso.

Neuquén, una de las 24 provincias argentinas, se ubica en el noroeste de la Patagonia y en 1995 tenía una población aproximada de 453000 habitantes (18). En los últimos tres decenios, el efecto de la inmigración, los adelantos en materia de educación, vivienda y saneamiento ambiental, y las acciones

CUADRO 1. Factores asociados con bajo peso al nacer (BPN) descritos más frecuentemente en la literatura médica

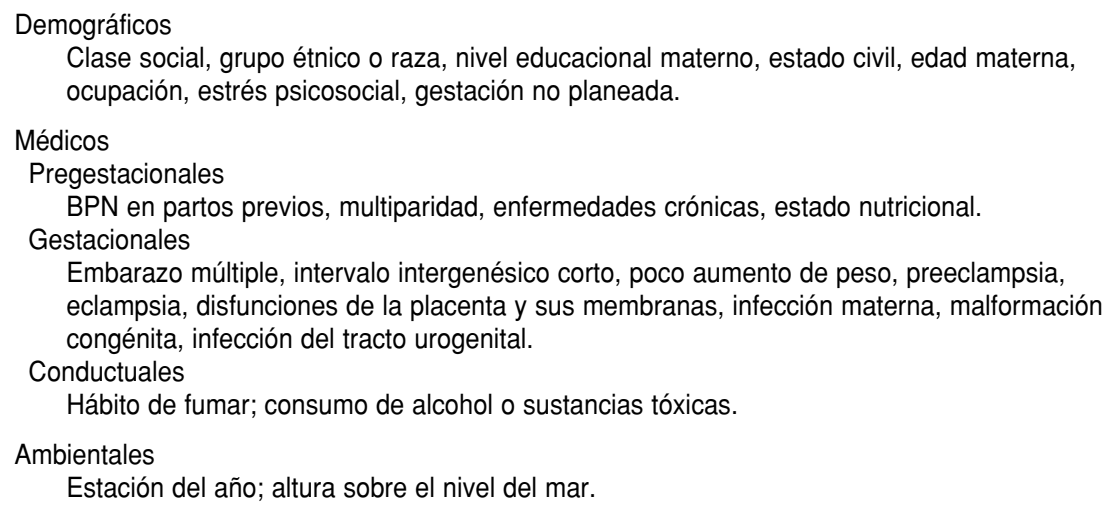

Clase social, grupo étnico o raza, nivel educacional materno, estado civil, edad materna, ocupación, estrés psicosocial, gestación no planeada.

\section{Médicos}

Pregestacionales

BPN en partos previos, multiparidad, enfermedades crónicas, estado nutricional.

Gestacionales

Embarazo múltiple, intervalo intergenésico corto, poco aumento de peso, preeclampsia, eclampsia, disfunciones de la placenta y sus membranas, infección materna, malformación congénita, infección del tracto urogenital.

Conductuales

Hábito de fumar; consumo de alcohol o sustancias tóxicas.

Ambientales

Estación del año; altura sobre el nivel del mar.

del Sistema Provincial de Salud han cambiado notablemente las estadísticas vitales de esta provincia. La tasa de mortalidad infantil se redujo de forma neta de 108,4 por 1000 en 1970, a 14,1 por 1000 en 1995 (18-23). Sin embargo, no se dispone de estimaciones confiables de la incidencia de BPN anteriores a 1986. El análisis de los certificados de nacimiento y de defunción de los períodos comprendidos entre 1986 y 1989 y entre 1990 y 1993 reveló una reducción sustancial de la tasa de mortalidad infantil (de 23,7 a 17,3 por 1000) y un mejoramiento de las tasas de supervivencia. No obstante estos logros y pese a que en ambos períodos estaba en marcha un programa estable de atención prenatal, no se han notificado cambios importantes de la tasa de incidencia de BPN.

En Neuquén existen dos registros o fuentes de datos sistemáticos sobre los nacidos vivos: el certificado de nacimiento (que es de base poblacional y que contiene poca información sobre posibles exposiciones o factores de riesgo) y el Sistema Informático Perinatal (SIP). El SIP es un sistema de registros desarrollado por el Centro Latinoamericano de Perinatología y Desarrollo Humano (CLAP) $(24,25)$. De acuerdo con las normas que estipula este sistema, durante la primera consulta prenatal para cada embarazada se llena un formulario que se actualiza en las consultas posteriores y que se completa durante la hospitaliza- ción, el parto y el puerperio. A partir de 1980 se comenzó a utilizar en la provincia una versión preliminar del SIP, que no se puso en funcionamiento hasta 1988. Entre 1988 y 1995, el SIP se actualizó en dos ocasiones. En las versiones posteriores del SIP se han incluido nuevas variables, como el aumento de peso, el número de cigarrillos fumados al día y el intervalo intergenésico. Actualmente se dispone de unos 46000 registros correspondientes al período de 1988 a 1995. Las principales ventajas de esta fuente de datos son las de proveer información sobre la mayoría de los factores de riesgo de $\mathrm{BPN}$ y de recolectar dicha información antes de conocerse el resultado del embarazo. Como desventajas cabe citar que los registros del SIP solo se llevan en los hospitales públicos (donde se atienden $70 \%$ de los partos de la provincia) y que los formularios no incluyen información sobre clase social, grupo étnico ni ocupación.

En el presente estudio se formularon dos hipótesis. Primero, la incidencia de niños con BPN es mayor en madres que no han tenido un contacto temprano y regular con los servicios de atención prenatal. Segundo, el efecto protector de estos servicios es más notable en las madres en riesgo de dar a luz hijos con BPN que en las demás. La investigación se llevó a cabo con los siguientes objetivos: conocer los factores de riesgo asociados con el BPN y su prevalencia en Neuquén, Argentina; 
CUADRO 2. Definición y categorías de las exposiciones consideradas en el estudio sobre el bajo peso al nacer. Neuquén, Argentina, 1988-1995

\begin{tabular}{|c|c|}
\hline Edad materna (años): & $\begin{array}{l}\text { Edad de la madre al comienzo de la gestación. (<17; 17-19; } \\
20-35 ; 36-40 ;>40) \text {. }\end{array}$ \\
\hline Nivel educativo: & $\begin{array}{l}\text { No asistió a la escuela; con primaria incompleta; con primaria } \\
\text { completa o secundaria incompleta; con secundaria completa o } \\
\text { estudios superiores. }\end{array}$ \\
\hline Estado civil: & Soltera; casada; unión estable; viuda o divorciada. \\
\hline $\begin{array}{l}\text { Antecedentes de bajo } \\
\text { peso al nacer: }\end{array}$ & Sí o no. \\
\hline Múltiples embarazos: & Más de tres embarazos previos: sí o no. \\
\hline Estado nutricional: & $\begin{array}{l}\text { El índice de masa corporal se usó como indicador del estado } \\
\text { nutricional materno y se calculó a partir de la talla y el peso antes } \\
\text { de la gestación. }(<20 ; 20-24 ; 25-29 ;>29) \text {. }\end{array}$ \\
\hline Intervalo intergenésico corto: & Parto previo en los últimos 18 meses: sí o no. \\
\hline $\begin{array}{l}\text { Hipertensión asociada con } \\
\text { el embarazo: }\end{array}$ & Preeclampsia o eclampsia durante el embarazo en curso: sí o no. \\
\hline Enfermedades crónicas: & Sí o no. \\
\hline $\begin{array}{l}\text { Anomalía o hemorragia de } \\
\text { placenta o sus membranas: }\end{array}$ & Sí o no. \\
\hline Infección del tracto urinario: & Sí o no. \\
\hline Otras infecciones: & Sí o no. \\
\hline Hábito de fumar: & Al comienzo de la gestación: sí o no. \\
\hline $\begin{array}{l}\text { Estación del año: } \\
\text { Atención prenatal: }\end{array}$ & $\begin{array}{l}\text { Parto en los meses de invierno (junio, julio y agosto): sí o no. } \\
\text { (Según las metas del programa de atención prenatal para la } \\
\text { provincia) }\end{array}$ \\
\hline $\begin{array}{l}\text { Momento de la primera } \\
\text { consulta prenatal: }\end{array}$ & $\begin{array}{l}\text { En el primer trimestre; durante los meses } 4 .^{\circ} \text { y } 5 .^{\circ} \text {; después del } \\
5 .^{\circ} \text { mes. }\end{array}$ \\
\hline $\begin{array}{l}\text { Número de consultas } \\
\text { prenatales: }\end{array}$ & Ninguna; $1-4 ;>4$ \\
\hline
\end{tabular}

analizar el efecto de la primera consulta prenatal y del número total de consultas durante el embarazo como factores protectores contra el BPN en mujeres con distinto grado de riesgo, y desarrollar una escala para identificar grupos en alto riesgo que sea aplicable al inicio de la gestación y que pueda utilizarse en actividades de prevención del BPN.

\section{MATERIALES Y MÉTODOS}

En el presente estudio, llevado a cabo del 25 de mayo de 1996 al 2 de septiembre de 1996, se utilizaron los datos del SIP y se empleó un diseño transversal que permitía estimar la frecuencia de factores de riesgo, era barato y rápido y estaba menos expuesto a sesgos de observación que otros estudios transversales, en que buena parte de la información sobre exposiciones se había obtenido antes de conocer el resultado final, es decir, el peso del niño al nacer. Este último se tomó como variable continua para estudiar su distribución, y también como variable binaria $(\mathrm{BPN}=$ peso al nacer menor de 2500 gramos). En el cuadro 2 aparecen las exposiciones consideradas en la investigación y sus categorías. Los restantes factores de riesgo potenciales (ocupación, clase social, grupo étnico, ingesta de alcohol, embarazo no planeado, etc.) no se incluyeron en el estudio, dado que en los formularios del SIP no se registran.

Debido al elevado número de registros disponibles, estos se dividieron en dos subarchivos empleando un método de asignación aleatoria computadorizada. El primer subarchivo - que contiene aproximadamente $50 \%$ de los datos (23105 registros) - se utilizó para el análisis y el segundo, para validar la escala de riesgo. Del estudio se excluyeron 391 embarazos múltiples $(1,7 \%)$ en virtud de su especial relación con el BPN. No se pudo conocer el peso al nacer de 68 recién nacidos $(0,3 \%)$, que fueron excluidos del análisis por conformar una baja proporción de casos. Hasta $5 \%$ de los registros carecían de toda la información necesaria sobre los factores de riesgo de interés para el estudio. Para conocer la posibilidad de que este hecho pudiese introducir un sesgo, se compararon las proporciones de los datos que faltaban sobre las mujeres que habían recibido atención adecuada y las que no la habían recibido, así como las proporciones de las que tuvieron hijos con BPN y sin BPN. Excepto en el caso del índice de masa corporal (IMC), no se encontraron diferencias significativas entre los grupos mencionados. Por ese motivo, y descartando la posibilidad de que se introdujera dicho sesgo, los registros sin información sobre algún factor de riesgo -exceptuado el IMC - se excluyeron del análisis final.

De los 23105 registros originales, se excluyeron los correspondientes a 391 embarazos múltiples, 68 registros que no contaban con información sobre el peso al nacer y 4180 que carecían de otros datos necesarios. Por consiguiente, en total se analizaron 18466 registros. Después de conocer la distribución del peso al nacer y la frecuencia de los factores de riesgo considerados, se estimaron las razones de posibilidades ( $\mathrm{RP} \mathrm{u}$ odds ratios en inglés) a fin de estimar la fuerza de la asociación entre dichos factores de riesgo y el BPN. Para analizar el efecto de posibles factores de confusión, se realizaron análisis estratificados. Por último, se construyeron modelos de regresión logística con el fin de estimar las RP ajustadas. La bondad de ajuste de estos modelos se analizó mediante la prueba de la razón de verosimilitudes.

Las RP estimadas por medio de los modelos de regresión logística se utilizaron para desarrollar la escala de riesgo de $\mathrm{BPN}$, que fue validada calculando el número de BPN esperados para cada puntaje de la escala en $50 \%$ de los registros restantes, así como comparándolo con el número de niños con BPN observados. Para realizar el 
CUADRO 3. Distribución de peso de los niños con bajo peso al nacer. Neuquén, Argentina, 1988-1995

\begin{tabular}{lrrr}
\hline Peso $(\mathrm{g})$ & No. & Porcentaje & $\begin{array}{r}\text { Porcentaje } \\
\text { acumulado }\end{array}$ \\
\hline$<500$ & 18 & 0,10 & 0,10 \\
$500-999$ & 150 & 0,81 & 0,91 \\
$1000-1499$ & 191 & 1,03 & 1,94 \\
$1500-1999$ & 307 & 1,67 & 3,61 \\
$2000-2499$ & 890 & 4,82 & 8,43 \\
$2500+$ & 6910 & 91,57 & 100,00 \\
Total & 18466 & 100,00 & 100,00 \\
\hline
\end{tabular}

análisis estadístico, se utilizó el programa STATA, versión 4.0.

\section{RESULTADOS}

La distribución del peso al nacer de los 18466 nacidos vivos mostró cierta curtosis hacia la izquierda, es decir, un exceso de niños de bajo peso (1556 con $\mathrm{BPN}$, equivalente a $8,43 \%$ ) (cuadro 3 ). La media del peso al nacer fue de 3208 g; la desviación estándar, de $599 \mathrm{~g}$ y los percentiles 5 y 95, de 2200 y $4050 \mathrm{~g}$, respectivamente. En el cuadro 4 figuran las frecuencias de los posibles factores de riesgo considerados en el estudio.

El análisis univariado y estratificado por número de consultas reveló RP brutas y ajustadas $\geq 1,5$ en mujeres con un número inadecuado de consultas prenatales, mujeres en los extremos de la edad reproductiva, solteras, con un IMC menor de 20, fumadoras, con un intervalo intergenésico breve, con preeclampsia o eclampsia, con hemorragia o anomalías de las membranas placentarias, o con hijos previos con BPN. Al excluir a las 6124 primigestas $(33,2 \%)$, la RP de las que habían tenido hijos con BPN aumentó de 2,26 a 3,07 (IC95\%: 2,63 a 3,60). Después de considerar que el verdadero efecto de este antecedente sería subestimado, las primigestas se incluyeron nuevamente en el análisis, puesto que su exclusión limitaría la posibilidad de generalizar los resultados.

En el cuadro 5 se presentan los resultados del modelo de regresión logística. La introducción de edad materna, estado civil, hábito de fumar, preeclampsia o eclampsia, antecedentes de hijos con BPN, hemorragia o anomalías de las membranas placentarias, número inadecuado de consultas prenatales, oportunidad de la primera consulta prenatal, IMC e intervalo intergenésico breve dio resultados significativos en pruebas de la razón de la verosimilitud. Los restantes factores en estudio - escolaridad, más de tres embarazos, infección del tracto urinario, enfermedades crónicas y parto en invierno- fueron excluidos del modelo. La inclusión tardía del IMC y del intervalo intergenésico por el menor número de registros con estos datos, no cambió de forma significativa las RP de los otros factores. La RP más elevada fue la correspondiente a la falta de atención prenatal ( $\mathrm{RP}=8,8$; IC95\%: 6,8 a 11,4). La fuerza de la asociación entre una atención prenatal inadecuada y tardía con el BPN también fue notable, y las RP de la hipertensión relacionada con el embarazo, hemorragias o anomalías de la pla-

CUADRO 4. Distribución de frecuencias de los factores de riesgo de bajo peso al nacer (BPN) considerados en el estudio. Neuquén, Argentina, 1988-1995

\begin{tabular}{|c|c|c|c|}
\hline Factor de riesgo & No. & Porcentaje & IC95\% \\
\hline \multicolumn{4}{|l|}{ Edad materna (años) } \\
\hline$<17$ & 1398 & 7,6 & 7,2 a 8,0 \\
\hline $17-19$ & 3366 & 18,2 & 17,6 a 18,7 \\
\hline 20-35 & 12395 & 67,1 & 66,4 a 67,8 \\
\hline $36-40$ & 1047 & 5,7 & 5,4 a 6,0 \\
\hline$>40$ & 260 & 1,4 & 1,2 a 1,6 \\
\hline \multicolumn{4}{|l|}{ Nivel educativo } \\
\hline Sin educación & 767 & 4,2 & 3,9 a 4,5 \\
\hline Escuela primaria incompleta & 3646 & 19,7 & 19,1 a 20,3 \\
\hline Escuela primaria completa, secundaria incompleta & 11452 & 62,0 & 61,3 a 62,7 \\
\hline Escuela secundaria completa & 2601 & 14,1 & 13,6 a 14,6 \\
\hline \multicolumn{4}{|l|}{ Estado civil } \\
\hline Casada & 6966 & 37,7 & 37,0 a 38,4 \\
\hline Unión estable & 8138 & 44,1 & 43,4 a 44,8 \\
\hline Viuda o divorciada & 200 & 1,1 & 0,9 a 1,7 \\
\hline Soltera & 3162 & 17,1 & 16,5 a 17,6 \\
\hline \multicolumn{4}{|l|}{ Número de consultas prenatales } \\
\hline Adecuado (>4) & 11775 & 63,8 & 63,1 a 64,5 \\
\hline Inadecuado (1-4) & 6102 & 33,0 & 32,3 a 33,7 \\
\hline Ninguna & 589 & 3,2 & 2,9 a 3,4 \\
\hline \multicolumn{4}{|l|}{ Oportunidad de la primera consulta prenatal } \\
\hline Antes del $4 .^{\circ}$ mes & 5532 & 30,0 & 29,3 a 30,7 \\
\hline Durante el $4 .^{\circ} \circ 5 .^{\circ}$ mes & 6460 & 35,0 & 34,3 a 35,7 \\
\hline Después del $5 .^{\circ}$ mes & 5885 & 31,9 & 31,3 a 32,6 \\
\hline Sin atención prenatal & 589 & 3,2 & 2,9 a 3,4 \\
\hline \multicolumn{4}{|l|}{ Otros factores } \\
\hline Antecedente de BPN & 1716 & 9,3 & 8,9 a 9,7 \\
\hline Multiparidad & 2957 & 16,0 & 15,5 a 16,5 \\
\hline Hábito de fumar & 2123 & 11,5 & 11,0 a 11,9 \\
\hline Infección del tracto urinario & 1084 & 5,9 & 5,6 a 6,2 \\
\hline Enfermedades crónicas & 93 & 0,5 & 0,4 a 0,6 \\
\hline Preeclampsia o eclampsia & 1564 & 8,5 & 8,1 a 8,9 \\
\hline Infección no urinaria & 282 & 1,5 & 1,3 a 1,7 \\
\hline \multicolumn{4}{|l|}{ Hemorragia o anomalía de la placenta o } \\
\hline sus membranas & 1591 & 8,6 & 8,2 a 9,0 \\
\hline Parto en invierno & 4701 & 25,5 & 24,9 a 26,1 \\
\hline Intervalo intergenésico breve & 1348 & 17,1 & 16,3 a 17,9 \\
\hline \multicolumn{4}{|l|}{ Índice de masa corporal } \\
\hline$<20$ & 1850 & 12,3 & 11,8 a 12,8 \\
\hline $20-24$ & 8704 & 57,8 & 57,0 a 58,6 \\
\hline $25-29$ & 3416 & 22,7 & 22,0 a 23,4 \\
\hline$\geq 30$ & 1080 & 7,2 & 6,8 a 7,6 \\
\hline
\end{tabular}


CUADRO 5. Razones de posibilidades (RP) ajustadas de bajo peso al nacer, junto con sus intervalos de confianza (IC95\%). Neuquén, Argentina, 1988-1995

\begin{tabular}{|c|c|c|}
\hline Factor de riesgo & $\mathrm{RP}$ & IC95\% \\
\hline \multicolumn{3}{|l|}{ Edad de la madre (años) $)^{a}$} \\
\hline$<17$ & 1,98 & 1,64 a 2,39 \\
\hline $17-19$ & 1,53 & 1,33 a 1,76 \\
\hline $36-40$ & 0,876 & 0,68 a 1,13 \\
\hline$>40$ & 1,70 & 1,16 a 2,48 \\
\hline \multicolumn{3}{|l|}{ Estado civil ${ }^{b}$} \\
\hline Unión estable & 1,07 & 0,94 a 1,22 \\
\hline Viuda o divorciada & 1,16 & 0,73 a 1,86 \\
\hline Soltera & 1,27 & 1,08 a 1,50 \\
\hline \multicolumn{3}{|l|}{ Otros factores } \\
\hline Antecedente de BPN & 2,45 & 2,11 a 2,86 \\
\hline Hábito de fumar & 1,41 & 1,20 a 1,65 \\
\hline Preeclampsia o eclampsia & 2,13 & 1,80 a 2,51 \\
\hline Hemorragia o anomalías de la placenta o sus membranas & 4,17 & 3,63 a 4,79 \\
\hline Intervalo intergenésico breve & 1,44 & 1,13 a 1,85 \\
\hline \multicolumn{3}{|l|}{ Índice de masa corporalc } \\
\hline$<20$ & 1,60 & 1,35 a 1,90 \\
\hline $25-29$ & 0,81 & 0,68 a 0,96 \\
\hline$\geq 30$ & 0,60 & 0,44 a 0,82 \\
\hline \multicolumn{3}{|l|}{ Número de consultas prenatales ${ }^{d}$} \\
\hline Inadecuado (1 a 4) & 1,89 & 1,65 a 2,16 \\
\hline Ninguna & 8,78 & 6,80 a 11,35 \\
\hline \multicolumn{3}{|l|}{ Oportunidad de la primera consulta prenatale } \\
\hline Durante el $4 .^{\circ} \circ 5 .^{\circ} \mathrm{mes}$ & 2,18 & 1,81 a 2,62 \\
\hline Después del $5 .^{\circ}$ mes & 2,93 & 2,40 a 3,57 \\
\hline
\end{tabular}

centa o sus membranas y el antecedente de haber tenido un hijo con BPN fueron mayores de 2 .

El efecto de una atención prenatal inadecuada y de la oportunidad de la primera consulta en la incidencia de BPN en 9289 mujeres con uno o más factores de riesgo "mayores" ( $\mathrm{RP}>$ 1,5) no mostró diferencias significativas comparado con el efecto en el grupo de bajo riesgo; sin embargo, pese a la superposición de los intervalos de confianza, se encontró que las razones de posibilidades fueron mayores en todas las categorías del grupo de alto riesgo, lo que indica una tendencia de mayor efecto en este grupo.

Con el propósito de obtener un modelo más simple y de fácil aplicación en la práctica médica diaria, se desarrolló una escala de riesgo, agrupando los factores de riesgo en tres categorías (1, 2 o 3 puntos) sobre la base de la magnitud de las RP estimadas mediante el modelo de regresión logística (cuadro 6). El número de consultas prenatales se excluyó de la escala, porque no se dispone de esta información durante la gestación. A (BPN). Neuquén, Argentina, 1988-1995 pesar de que ciertos datos no pueden obtenerse durante la primera consulta, es posible actualizar la escala en consultas posteriores.

Para analizar la asociación entre los puntajes de la escala y los de la variable de resultado (BPN), se estimaron los riesgos relativos (RR) para cada puntaje, tomando como grupo de referencia a las mujeres con un puntaje de cero (es decir, sin factores de riesgo). Los resultados de este análisis revelaron la existencia de una relación directa entre los puntajes obtenidos en la escala y los RR estimados: el RR de las mujeres con una puntuación de 1 en la escala fue de 1,62 ; el de las mujeres con una puntuación de 9 fue de 18,5 (cuadro 7).

Para validar la escala, se usaron $50 \%$ de los datos que habían sido excluidos del análisis por asignación aleatoria. En ellos se calculó la incidencia de bajo peso esperada por grupo de puntaje y su distribución se comparó con la de los pesos al nacer observados. Para realizar estos cálculos, se utilizaron el riesgo del grupo de referencia $(0,0213)$ y el intervalo de confianza de $95 \%$ de los RR correspondientes a cada puntuación de la escala. Como se observa en el cuadro 8 y en la figura 1, el número de niños que tuvieron BPN se encontró dentro del recorrido de los valores esperados para cada uno de los puntajes en la escala. Por último, los 21762 registros utilizados en la valida-

CUADRO 6. Escala aditiva para predecir el riesgo de tener un hijo con bajo peso al nacer

\begin{tabular}{|c|c|c|c|}
\hline \multirow[b]{2}{*}{ Factor de riesgo } & \multicolumn{3}{|c|}{ Categorías de riesgo } \\
\hline & 1 & 2 & 3 \\
\hline Edad materna (años) & $17-19$ ó >40 & $<17$ & \\
\hline Estado civil & Soltera & & \\
\hline Antecedente de BPN & & Hijo previo con BPN & \\
\hline $\begin{array}{l}\text { Hábito de fumar } \\
\text { Índice de masa }\end{array}$ & Fumadora & & \\
\hline corporal & $<20$ & & \\
\hline Intervalo intergenésico & $<18$ meses & & \\
\hline $\begin{array}{l}\text { Preeclampsia } \\
\text { o eclampsia }\end{array}$ & & $\begin{array}{c}\text { Preeclampsia } \\
\text { o eclampsia }\end{array}$ & \\
\hline $\begin{array}{l}\text { Hemorragia o anomalía } \\
\text { de la placenta o sus } \\
\text { membranas }\end{array}$ & & & $\begin{array}{l}\text { Hemorragia o anomalía } \\
\text { de la placenta o sus } \\
\text { membranas }\end{array}$ \\
\hline $\begin{array}{l}\text { Oportunidad de la primera } \\
\text { consulta prenatal }\end{array}$ & & $4 .^{\circ} \circ 5^{\circ} \mathrm{mes}$ & Después del $5 .^{\circ}$ mes \\
\hline
\end{tabular}


CUADRO 7. Asociación entre el puntaje en la escala y el riesgo de dar a luz un niño con bajo peso al nacer (BPN). ${ }^{a}$ Neuquén, Argentina, 1988-1995

\begin{tabular}{crrrrr}
\hline Escala & BPN (No.) & Total & Riesgo & $R R$ & IC95\% ${ }^{\mathrm{b}}$ \\
\hline 0 & 53 & 2489 & 0,0213 & - & - \\
1 & 46 & 1331 & 0,0346 & 1,62 & 1,10 a 2,40 \\
2 & 254 & 5318 & 0,0478 & 2,24 & 1,67 a 3,00 \\
3 & 255 & 3362 & 0,0758 & 3,56 & 2,66 a 4,77 \\
4 & 288 & 2748 & 0,1048 & 4,92 & 3,69 a 6,56 \\
5 & 220 & 1397 & 0,1575 & 7,40 & 5,52 a 9,91 \\
6 & 177 & 923 & 0,1918 & 9,01 & 6,69 a 12,13 \\
7 & 116 & 469 & 0,2473 & 11,62 & 8,52 a 15,83 \\
8 & 78 & 254 & 0,3071 & 14,42 & 10,43 a 19,94 \\
$9+$ & 69 & 175 & 0,3943 & 18,52 & 13,40 a 25,59 \\
Total & 1556 & 18466 & 0,0843 & & \\
\hline
\end{tabular}

a Prueba no paramétrica de tendencia en los distintos puntajes en la escala $\left(\chi^{2}=962, P<0,0001\right)$

b IC95\%: intervalo de confianza de $95 \%$.
FIGURA 1. Comparación entre el número de niños con bajo peso al nacer (BPN) observados y esperados, según la escala de riesgo. Neuquén, Argentina, 1988-1995

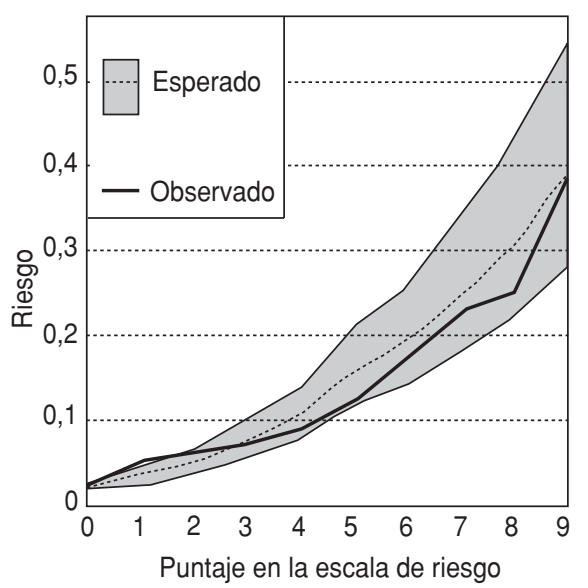

las enfermedades crónicas y de la estación del año en que se tuvo el parto no produjo mejorías estadísticas significativas cuando se aplicó el modelo de regresión logística. A pesar de que solo $2,85 \%$ de las mujeres estudiadas carecieron por completo de atención prenatal, únicamente un tercio acudió a la primera consulta durante el primer trimestre del embarazo.

Se confirmó, por otra parte, que la proporción de mujeres que recibieron una atención prenatal adecuada y oportuna fue más elevada en el grupo de mayor escolaridad. Es sorprendente, sin embargo, que una buena fracción de estas mujeres haya recibido una atención prenatal inadecuada. La proporción de mujeres que acudieron a un número adecuado de consultas prenatales también fue más alta entre las que habían tenido infecciones del tracto urinario, enfermedades crónicas y preeclampsia o eclampsia, pero con la única excepción de las mujeres con infecciones del tracto urinario, estos factores no se asociaron con un inicio más oportuno de la atención prenatal. Por otro lado, y al contrario de lo que se esperaba, la proporción de mujeres que recibieron una atención prenatal inadecuada o que acudieron tarde a la primera consulta prenatal fue mayor entre las que habían tenido un hijo con BPN que entre 
FIGURA 2. Distribución del peso al nacer en cada grupo de la escala de riesgo. ${ }^{a}$ Neuquén, Argentina, 1988-1995

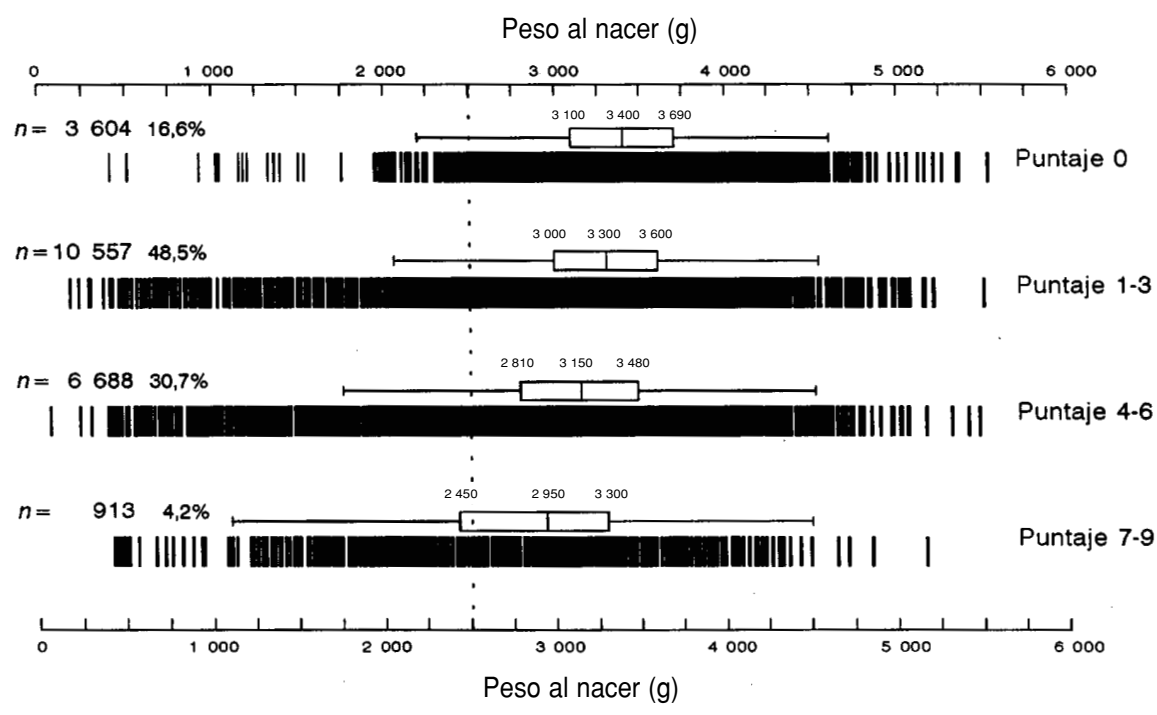

a Peso promedio al nacer correspondiente al grupo de referencia (puntaje de cero) $=3421 \mathrm{~g}$. El análisis de regresión lineal reveló una disminución promedio de $168 \mathrm{~g}$ del peso al nacer en cada grupo sucesivo de la escala de riesgo. $F(1,21760)=$ 1114,$02 ; P<0,0001$.

las que no tenían ese antecedente. Este es uno de los resultados más importantes del estudio, más aún cuando se tiene en cuenta que la RP de BPN de las que ya habían tenido un hijo con BPN aumentó de 2,6 a 3,07 al excluirse a las primigestas del análisis.

La disponibilidad de una escala que permita identificar a mujeres con distintos riesgos de tener un hijo con $\mathrm{BPN}$ puede ser muy útil a la hora de idear estrategias para reducir el riesgo de $\mathrm{BPN}$, especialmente en vista de que la atención prenatal parece conferir mayor protección a mujeres en riesgo de tener hijos con BPN que en mujeres sin ningún factor de riesgo. A pesar de la relativa sencillez de la escala aditiva que hemos creado, al compararse con escalas que se basan en modelos multiplicativos, pudimos demostrar la relación lineal entre los puntajes y el riesgo de dar a luz un niño con $\mathrm{BPN}$.

Resulta evidente, dada la relación que muestran la edad materna y el estado civil con la incidencia de BPN, que un objetivo de capital importancia en la reducción del BPN consiste en reducir la frecuencia del embarazo en jóvenes solteras. Asimismo, los responsables de planificar los servicios de salud deben tener presente el papel importante que desempeñan el tabaquismo, el intervalo intergenésico y el estado nutricional en el riesgo que corren las mujeres en edad reproductiva de tener un hijo con BPN.

Aunque fue pequeño el porcentaje de mujeres que no recibieron ninguna atención prenatal, solo $30 \%$ tuvo su primer contacto con los servicios de atención prenatal en el primer trimestre del embarazo. Si bien es cierto que la falta de atención prenatal no puede considerarse como un factor causal de $\mathrm{BPN}$, la diferencia entre el riesgo atribuible poblacional de BPN entre mujeres cuyo riesgo es alto y bajo $(49,3 \%$ y $68,5 \%$, respectivamente) permite concluir que si se aumenta la proporción de mujeres que reciben atención prenatal oportuna se logrará reducir notablemente la frecuencia de BPN. Cabe afirmar, sobre la base de los resultados obtenidos en este estudio, que nuestra escala de riesgo representa un instrumento clave para utilizar acertadamente los recursos disponibles y aplicar con eficiencia las estrategias destinadas a reducir el riesgo de BPN. Falta investigar por qué tantas mujeres no acuden a los servicios de atención prenatal en los primeros meses de la gestación y por qué son relativamente pocas las que buscan atención prenatal a pesar de haber tenido un hijo previo con BPN.

\section{REFERENCIAS}

1. World Health Organization. Expert Group on Prematurity: final report. Geneva: WHO; 1950. (Technical Report 27)

2. World Health Organization. Manual of the International Classification of Diseases. Adapted 1U48. Geneva: WHO; 1950.

3. World Health Organization. International Classification of Diseases. 9th revision. Geneva: WHO; 1977
4. McCormick MC. The contribution of low birth weight to infant mortality and childhood mortality. N Engl J Med 1985;312:82-90.

5. McCormick MC, Brooks-Gunn J, Shorter T, Holmes JH, Wallace CY, Heagarty MC. Outreach as case finding: its effect on enrollment in prenatal care. Med Care 1989;27:103-111.

6. Parazzini F, Pirrotta N, La Vecchia C, Bocciolone L, Fedele L. Determinants of perinatal and infant mortality in Italy. Rev Epidemiol Sante Publique 1992;40:15-24.

7. Overpeck MD, Hoffman HJ, Prager K. The lowest birth-weight infants and the US infant mortality rate: NCHS 1983 linked birth/infant death data. Am J Public Health 1992;82: 441-444.

8. Shapiro S, Unger J. Relation of weight at birth a cause of death and age at death in the neo- 
natal period: United Sates, early 1950. Vital Stat Special Rep 1954;39:226-299.

9. Jacob J. Cost-saving of regionalized perinatal care for low birthweight infants. Alaska Med 1986;28:53-56.

10. Tudehope DI, Lee W, Harris F, Addison C. Cost-analysis of neonatal intensive and special care. Aust Paediatr J 1989;25:61-65.

11. Sandhu B, Stevenson RC, Cooke RW, Pharoah PO. Cost of neonatal care for very-low-birthweight infants. Lancet 1986;15:600-603.

12. Robert L, Goldberg MD. The prevention of low birthweight and its sequelae. Prev Med 1994;3:627-631.

13. Barker DJP, Glockman PD, Godfrey KM, Harding JE, Owens JA, Robinson JS. Fetal nutrition and cardiovascular disease in adult life. Lancet 1993;341:938-941.

14. Rose G. The strategy of preventive medicine. New York: Oxford University Press; 1992.

15. Villar J, Belizan JM. The relative contribution of prematurity and fetal growth retardation to low birth weight in developing and developed societies. Am J Obstet Gynecol 1982;143: 793-798.
16. World Health Organization. The World Health Report: WHO's contributions to World Health. Geneva: WHO; 1995. pp. 77-79.

17. Pérez Escamilla R, Pollitt E. Causas y consecuencias del retraso del crecimiento intrauterino en América Latina. Bol Oficina Sanit Panam 1992;112:473-493.

18. Neuquén, Argentina, Subsecretaría de Salud del Neuquén. Estadísticas vitales 1995. Neuquén: Subsecretaría de Salud del Neuquén; 1996.

19. Neuquén, Argentina, Subsecretaría de Salud del Neuquén. Estadísticas vitales 1985-1988. Neuquén: Subsecretaría de Salud del Neuquén; 1989.

20. Neuquén, Argentina, Subsecretaría de Salud del Neuquén. Estadísticas vitales 1989-1991. Neuquén: Subsecretaría de Salud del Neuquén; 1992.

21. Neuquén, Argentina, Subsecretaría de Salud del Neuquén. Estadísticas vitales 1992. Neuquén: Subsecretaría de Salud del Neuquén; 1993.

22. Neuquén, Argentina, Subsecretaría de Salud del Neuquén. Estadísticas vitales 1993. Neu- quén: Subsecretaría de Salud del Neuquén 1994

23. Neuquén, Argentina, Subsecretaría de Salud del Neuquén. Estadísticas vitales 1994. Neuquén, Argentina, Subsecretaría de Salud del Neuquén; 1995.

24. Centro Latinoamericano de Perinatología y Desarrollo Humano (CLAP), Organización Panamericana de la Salud. Sistema Informático Perinatal. Montevideo: OPS; 1993. (Publicación científica CLAP No. 1203).

25. Centro Latinoamericano de Perinatología y Desarrollo Humano (CLAP), Organización Panamericana de la Salud. Procesamiento de datos del Sistema Informático Perinatal: Centro Latinoamericano de Perinatología y Desarrollo Humano. Montevideo: OPS; 1993. (Publicación científica CLAP No. 1207).

Manuscrito recibido el 19 de mayo de 1997 y aceptado para publicación en versión revisada el 15 de enero de 1998.

ABSTRACT Low birthweight (LBW) is the main known determinant of infant mortality. In spite of the sharp decrease in infant mortality rates and of the rise in survival rates for children with LBW, no important decrease in LBW rates has been observed in Neuquen,

Risk factors for low birthweight
Argentina. The purpose of this study was to try to understand the risk factors for LBW, the frequency of LBW in the population, and the role of prenatal care in its prevention, as well as to develop a risk factor scale that could be used to identify women at higher risk of giving birth to a child with LBW. With this in mind we performed a cross-sectional study based on $50 \%$ of the data entered into the Perinatal Information System for $1988-1995$ by the 29 hospitals in Neuquen province (46 171 births). The distribution of birthweight and the frequency of potential risk factors for LBW were examined. The relationship between such factors and LBW was studied using a logistic regression model. On the basis of the results obtained, an additive scale was drawn up and validated with the remaining $50 \%$ of the data for registered births. The highest odds ratio (OR) was seen in women who had no prenatal care $(\mathrm{OR}=8,78 ; 95 \% \mathrm{CI}$ : $6,7$ to 11,4$)$. ORs for inadequate prenatal care, lateness in attending the first prenatal visit, preeclampsia or eclampsia, hemorrhage and anomalies of the placenta or placental membranes, and a history of a previous child with LBW were greater than 2,0 . The risk of having children with LBW was also higher in women over the age of 40 , women under 20, single women, smoking mothers, women with an intergenesic interval of less than 18 months, and women with a body mass index of less than 20. Finally, there was a direct linear relationship between points on the risk scale and the risk of having a LBW infant. 